\title{
Neonatal pneumonia in a rural primary care hospital in Bangladesh: prevalence, validation of clinical features and their outcome
}

Md. Nazim Uzzaman ${ }^{1}$, Md. Al Fazal Khan², Tahmeed Ahmed³, Mohammad Habibur Rahman Sarker ${ }^{4}$, Sultana Yeasmin $^{5}$, Kazi Nazmus Saqeeb ${ }^{6}$, Mohammod Jobayer Chisti ${ }^{7}$

\begin{abstract}
Objective: To explore prevalence, validity of associated factors and their outcome of pneumonia in neonates.

Methods: We retrospectively enrolled neonates admitted to a rural hospital in Bangladesh from January 2012-December 2014. Those with pneumonia constituted cases $(n=142)$ and randomly selected three folds of cases from those without pneumonia formed the controls $(n=426)$. Pneumonia was diagnosed by hospital physicians based on respiratory difficulty and/or abnormal auscultatory findings in lungs.

Result: The deaths were significantly higher among the cases than the controls $(p=0.025)$. In logistic regression analysis, fast breathing, lower chest wall in-drawing, adventitious sound and cough were independently associated with neonatal pneumonia (for all, $p<0.01$ ). However, best of all, sensitivity of fast breathing and lower chest wall in-drawing was $94 \%$ and $76 \%$ and specificity $81 \%$ and $82 \%$ respectively.
\end{abstract}

Conclusion: The results underscore the importance of adherence to WHO defined clinical signs in diagnosing pneumonia in neonates especially in resource limited settings.

Key Words: Bangladesh; fast breathing; lower chest wall in-drawing; pneumonia; neonate;

\section{Introduction:}

Globally, there is substantial gain in child survival over the past two decades. However, improvement is seen uneven both

1. Dr. Md Nazim Uzzaman, Medical Officer, Nutrition and Clinical Services Division (NCSD), International Centre for Diarrhoeal Disease Research, Bangladesh (icddr,b), Dhaka, Bangladesh

2. Dr. Md Al Fazal Khan, Associate Scientist and Head, Matlab Hospital, NCSD, icddr,b, Dhaka, Bangladesh

3. Dr. Tahmeed Ahmed, Senior Scientist and Senior Director, NCSD, icddr,b, Dhaka, Bangladesh

4. Dr. Mohammad Habibur Rahman Sarker, Medical Officer, NCSD, icddr,b, Dhaka, Bangladesh

5. Dr. Sultana Yeasmin, Senior Medical Officer, NCSD, icddr,b, Dhaka, Bangladesh

6. Dr. Kazi Nazmus Sakib, Medical Officer, Matlab Hospital, NCSD, icddr,b, Dhaka, Bangladesh

7. Dr. Mohammod Jobayer Chisti, Scientist, NCSD \& Clinical Lead, Intensive Care Unit \& Head, Clinical Research, Hospitals, icddr,b; Dhaka, Bangladesh

Corresponding Author:

Dr. Mohammod Jobayer Chisti

MBBS, MMed, PhD

Scientist, NCSD \& Clinical Lead

Intensive Care Unit \&

Head, Clinical Research, Hospitals, icddr,b

68 Shaheed Tajuddin Ahmed Sarani, Mohakhali

Dhaka 1212, Bangladesh

Email: chisti@icddrb.org across and within countries. ${ }^{1}$ It is anticipated that 2.7 million of the 5.9 million deaths in children yearly throughout the world occur in the first 28 days of life which is $45 \%$ of under-five child mortality. ${ }^{2}$ Pneumonia is still remaining as one of the leading causes of neonatal illness that require hospitalization and accounts for $5 \%$ of global neonatal deaths, most of whom occur in developing countries. ${ }^{3}$ In Bangladesh, neonatal illnesses account for $60 \%$ of under-five child mortality of which neonatal pneumonia is responsible for $2 \%$ deaths. ${ }^{4}$ In a resource poor setting hospital, it is often difficult to diagnose pneumonia using laboratory investigations such as chest radiograph, blood culture, or chest ultrasonography ${ }^{5}$, although, World Health Organization (WHO) recently recommended at least oxygen saturation should be measured using pulse oximetry. ${ }^{6}$ Therefore, it is very important to understand the clinical predicting factors of neonatal pneumonia that may help in early diagnosis and effective management in order to improve the prognosis of patients with neonatal pneumonia. Although, a lot of efforts have already been made to describe this important issue, more work in resource poor settings should be continued to improve the knowledge to curve further deaths in neonatal pneumonia.

In Matlab hospital of the International Centre for Diarrhoeal Disease Research, Bangladesh (icddr,b), as in other hospitals of Bangladesh, neonates often present with prematurity, birth asphyxia, sepsis, pneumonia, diarrhea and other comorbidities. However, there is limited published data on prevalence, clinical predicting factors of neonatal pneumonia and their outcome. The objectives of the study were to 
identify the prevalence, clinical predictors and outcome of neonatal pneumonia in Matlab hospital of icddr,b.

\section{Methods:}

\section{Study design}

It was a retrospective chart analysis where we enrolled all the neonates ( 0 to 28 days of life) who were admitted to neonatal ward of Matlab hospital, icddr,b from 01 January 2012 to 31 December 2014. Neonatal pneumonia was diagnosed by the treating physicians of the hospital based on respiratory difficulty and/or abnormal auscultatory finding in lungs. This was designated as 'Doctor's diagnosis'.? Randomly selected three folds of neonatal pneumonia from those without pneumonia constituted the comparison group. Verbal consent was obtained from the parents or guardians of all participating neonate and the study was approved by the Ethical Review Committee of icddr,b.

\section{Setting}

Since 1966, icddr,b has been maintaining one of the richest, most comprehensive and longest running, longitudinal data resources in the developing world, producing regular accurate demographic and health data for rural Bangladesh in the icddr,b's field site at Matlab. The Health and Demographic Surveillance System at Matlab covers a population of about 225,000 which provides data necessary to plan, conduct, and evaluate various types of public-health intervention researches in Bangladesh. In addition, Matlab Hospital of icddr,b provides clinical services to the population of its service area and beyond. Each year this hospital provides treatment of around 48000 patients. It has two distinct units. One is diarrheoa treatment unit which deals with diarrhoea, with or without associated complications or other health problems. Another is maternal and child health care unit which deals with neonates, children of under five years of age and women of reproductive age from its service area.

\section{Patient management}

Neonates admitted to the Neonatal Ward receive oxygen therapy, antibiotics, and supportive care including intravenous fluids, frequent monitoring and nutritional support (breast milk, micronutrients etc). All children in the study were seen by the attending physician, a history was taken and clinical examination was performed. Arterial oxygen saturation $(\mathrm{SpO} 2)$ was measured using a portable pulse oximeter (OxiMax N-600; Nellcor, Boulder, CO, USA). Neonates with hypoxaemia received $\mathrm{O}_{2}$ supplementation through nasal prongs $(0.5 \mathrm{l} / \mathrm{min})$. Antibiotics were given to neonatal pneumonia following WHO algorithm. ${ }^{6}$

\section{Measurements}

Case report forms were developed, pre-tested and finalized for data acquisition. Characteristics analyzed were gender, age, cough, fast breathing, grunting respiration, bronchial breath sound, adventitious sound, lower chest wall indrawing, cyanosis, abdominal distension, anaemia, jaundice, congenital anomaly, hypoxaemia, severe wasting, temperature, hospital stay and outcome. The important parameters are shown in Table 1.
Table 1: Definition of important clinical study parameters

\begin{tabular}{ll}
\hline Parameters & Definition \\
\hline Clinical pneumonia & $\begin{array}{l}\text { Respiratory difficulty and/or abnormal } \\
\text { auscultatory findings in lungs }\end{array}$ \\
Lower chest wall in-drawing & $\begin{array}{l}\text { Inward movement of the bony structures } \\
\text { of the lower chest wall with inspiration }\end{array}$ \\
Hypoxaemia & $\begin{array}{l}\text { If SPO2 without O2 is }<90 \% \\
\text { Severe wasting } \\
\text { median value of the NCHS }\end{array}$ \\
Radiological pneumonia & Other (non-end-point) infiltrate \\
Added sound in lungs & Wheeze \pm Crackle \\
\hline
\end{tabular}

\section{Analysis}

All data were entered into SPSS for Windows (version 17.0; SPSS Inc, Chicago) and Epi Info 2000 (version 6.0; USD, Stone Mountain, GA). Differences in proportions were compared by Chi-square test or Fisher's exact test as appropriate. In normally distributed data, differences in means were compared by Student's t-test, and in non-normally distributed data, differences in median were compared by Mann-Whitney test. A probability of $<0.05$ was considered statistically significant. Strength of association was determined by calculating odd ratio (OR) and their $95 \%$ confidence intervals (CI). In analyzing the independent predicting factors of neonatal pneumonia, variables were initially analyzed in a univariate model, and then covariates were adjusted for using logistic regression. The sensitivity, specificity, positive predictive value and negative predictive value of the independent predictors of neonatal pneumonia were also calculated.

\section{Results:}

The prevalence of neonatal pneumonia at Matlab Hospital, icddr,b during the study period was estimated to be $9 \%$ $(142 / 1544)$. The median age of the neonates with pneumonia and their comparison groups (without pneumonia) were 15 days (IQR: 10,22$)$ and 6 days (IQR: 1,15$)$ respectively and $95(67 \%)$ of the cases and $239(56 \%)$ of the controls were males. The proportion of neonates who had grunting respiration, bronchial breath sound, cyanosis, abdominal distension, anaemia, jaundice, congenital anomaly, temperature and severe wasting were comparable between groups (Table 1). The case fatality was significantly higher among neonates who had pneumonia compared to those without pneumonia and their duration of hospital stay was often higher than their counterparts (Table 2). Only 11 chest $\mathrm{x}$-rays were available and all of them were positive for pneumonia. In logistic regression analysis, after adjusting for potential confounders such as cough, fast breathing, hypoxaemia, adventitious sound in lungs, lower chest wall in-drawing and age at admission were retained as independent predictors of neonatal pneumonia (Table 3). Sensitivity for cough, fast breathing, hypoxaemia, adventitious sound in lungs, lower chest wall in-drawing compared to doctor's diagnosis of neonatal pneumonia were $48 \%, 94 \%, 8 \%, 49 \%$, $76 \%$ and their specificity were $94 \%, 81 \%, 97 \%, 96 \%, 82 \%$ respectively (Table 4). 
Bangladesh Crit Care J September 2016; 4 (2): 74-78

Table 2: Characteristics of the neonates with and without pneumonia admitted in the neonatal ward of the Matlab Hospital of icddr,b

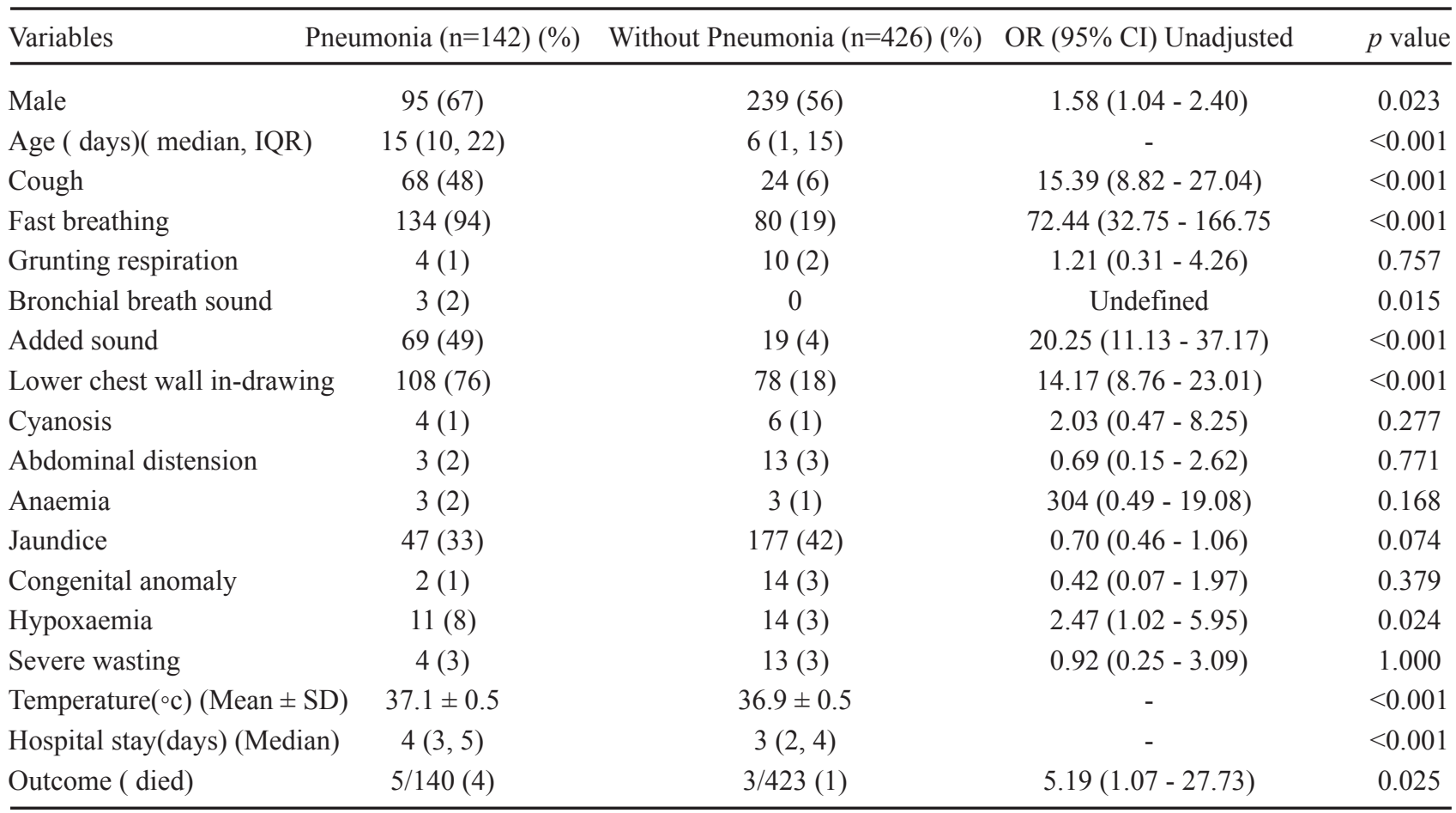

OR, Odds ratio; CI, Confidence interval; SD, Standard deviation; IQR, Interquartile range.

Table 3: Results of logistic regression to explore the independent predictors of pneumonia among the admitted neonates in Neonatal ward of the Matlab Hospital of icddr,b

\begin{tabular}{lccc}
\hline Characteristics & Adjusted OR & $95 \%$ CI & $p$ value \\
\hline Male & 1.54 & $0.77-3.09$ & 0.223 \\
Cough & 11.01 & $4.28-28.31$ & $<0.001$ \\
Fast breathing & 45.22 & $17.58-116.33$ & $<0.001$ \\
Hypoxaemia & 4.01 & $1.02-15.80$ & 0.047 \\
Added sound & 4.18 & $1.79-9.72$ & 0.001 \\
Lower chest wall indrawing & 3.18 & $1.52-6.65$ & 0.002 \\
Age in days (Median, IQR) & 0.92 & $0.89-0.97$ & $<0.001$ \\
CI, confidence interval. & & & \\
\hline
\end{tabular}

Table 4 Sensitivity, specificity, positive and negative predictive value of predictors of neonatal pneumonia

\begin{tabular}{lcccccc}
\hline Variable & $\begin{array}{c}\text { Pneumonia } \\
(\mathrm{n}=142) \\
\mathrm{n}(\%)\end{array}$ & $\begin{array}{c}\text { Without } \\
\text { Pneumonia } \\
(\mathrm{n}=426) \mathrm{n}(\%)\end{array}$ & $\begin{array}{c}\text { Sensitivity } \\
(95 \% \mathrm{CI})\end{array}$ & $\begin{array}{c}\text { Specificity } \\
(95 \% \mathrm{CI})\end{array}$ & $\begin{array}{c}\text { Positive } \\
\text { predictive } \\
\text { value } \\
(95 \% \mathrm{CI})\end{array}$ & $\begin{array}{c}\text { Negative } \\
\text { predictive } \\
\text { value } \\
(95 \% \mathrm{CI})\end{array}$ \\
\hline Cough & $68(48)$ & $24(6)$ & $48(39-56)$ & $94(92-96)$ & $74(65-82)$ & $84(81-88)$ \\
Fast breathing & $134(94)$ & $80(19)$ & $94(89-97)$ & $81(77-85)$ & $63(56-69)$ & $98(95-99)$ \\
Hypoxaemia & $11(8)$ & $14(3)$ & $8(4-14)$ & $97(94-98)$ & $44(25-65)$ & $76(72-79)$ \\
Added sound & $69(49)$ & $19(4)$ & $49(40-57)$ & $96(93-97)$ & $78(68-86)$ & $85(81-88)$ \\
Lower chest wall in-drawing & $108(76)$ & $78(18)$ & $76(68-83)$ & $82(77-85)$ & $58(51-65)$ & $91(88-94)$ \\
CI, confidence interval. & & & & & & \\
\hline
\end{tabular}




\section{Discussion:}

The clinical diagnosis of neonatal pneumonia is often subjective and unreliable. ${ }^{8}$ A lot of efforts have been taken to define neonatal pneumonia without proper validation. ${ }^{9}$ The optimal diagnosis of pneumonia relies on a combination of history, clinical signs and chest X-ray. ${ }^{10,11}$ While chest X-rays are generally regarded as a reliable diagnostic tool in all forms of pneumonia, variations in inter-observer interpretation are often intriguing. ${ }^{12-14}$ Despite the presence of clinical signs of pneumonia, the radiographic changes may be vague or inconclusive or even absent. ${ }^{15}$ On the other hand, clinical signs of pneumonia can be absent in the presence of radiological signs of pneumonia. ${ }^{16,17}$ Moreover, adequate laboratory and radiological services are frequently not available in primary health care facilities where neonates present with pneumonia. Auscultation performed by primary healthcare workers in resource-poor settings has not been sufficiently validated as a diagnostic tool in pneumonia. Given these limitations, the WHO recommends that the diagnosis of pneumonia should primarily be based on visible clinical parameters, including respiratory rate and chest wall in-drawing. ${ }^{18-20}$ However, for practical purposes, WHO does not distinguish neonatal pneumonia from other forms of neonatal infection such as neonatal sepsis because of overlapping in their clinical signs. ${ }^{21}$

Our observation of good sensitivity and specificity of fast breathing and lower chest wall in-drawing in neonates are very promising. The observed validity of these two parameters is quite robust compared to those observed in children 0 - 6 years. ${ }^{22}$ The findings of our study reveal that fast breathing ( $\geq 60$ per minute) is the most sensitive predictors of neonatal pneumonia with a high sensitivity of $94 \%$ and specificity of $81 \%$. We also observed that lower chest wall in-drawing has been revealed as one of the significant predictors of pneumonia having sensitivity and specificity of $76 \%$ and $86 \%$ respectively. In a previous study on $0-5$ years of children, the sensitivity of lower chest wall in-drawing was $71 \%$ but the specificity was low to $59 \%{ }^{23}$. Although, cough and adventitious sound in lungs on auscultation had poor sensitivity, they carried almost same importance with specificity. Hypoxaemia also showed very poor sensitivity but very high specificity which indicates that once a neonate has hypoxemia, in $97 \%$ cases the neonate will have pneumonia, therefore, this important parameter needs to be measured in all neonates requiring hospitalization. To our knowledge, our study is the first one which validated the clinical signs of neonatal pneumonia. The results re-confirm the high validity of WHO defined clinical signs in diagnosing pneumonia in hospitalized neonates.

The higher case fatality in neonatal pneumonia compared to those without pneumonia in our study population is understandable and consistent with global scenario of neonatal deaths. ${ }^{2}$

The limitation of the study is the small sample size, lack of radiologic evidence for all the cases and wide confidence intervals, and bias related to the subjectivity in the assessment of the clinical signs of pneumonia in neonates. The another limitation is the retrospective nature of our study, which confines our sample size, and did not allow for the systematic collection of a broader range of features of potential interest, or for focus on selected clinical features.

In conclusion, based on results of our data, we may conclude that neonates who have history of cough, present with fast breathing, lower chest wall in-drawing, and adventitious sound in lungs are likely to have pneumonia and the results underscore the importance of relying on WHO defined clinical signs for the diagnosis of neonatal pneumonia especially in resource poor clinical settings, that may help healthcare providers in early diagnosis of neonatal pneumonia and thereby reduce mortality in early infancy.

\section{Funding:}

This work was supported by core donors which provide unrestricted support to icddr,b for its operations and research. Current donors providing unrestricted support include: Government of the People's Republic of Bangladesh; the Department of Foreign Affairs, Trade and Development (DFATD), Canada; Swedish International Development Cooperation Agency (Sida) and the Department for International Development (UK Aid).

\section{Acknowledgement:}

We gratefully acknowledge the donors for their support to icddr,b's research efforts. We would like to express our sincere thanks to all physicians, nurses, members of the feeding team and cleaners of Neonatal Ward for precious support and contribution during patient enrollment and data collection. We would also like to convey our gratitude to care-givers, mothers of the study participants for their consent to enroll their babies in the study.

\section{Competing Interest:}

All authors have declared that no competing interests exist.

\section{References:}

1. WHO/UNICEF. Ending Preventable Child Deaths from Pneumonia and Diarrhoea by 2025. The Integrated Global Action Plan for the Prevention and Control of Pneumonia and Diarrhoea (GAPPD). . 2013.

2. Liu L, Johnson HL, Cousens S, et al. Global, regional, and national causes of child mortality: an updated systematic analysis for 2010 with time trends since 2000. Lancet 2012; 379(9832): 2151-61.

3. Wardlaw T, You D, Hug L, Amouzou A, Newby H. UNICEF Report: enormous progress in child survival but greater focus on newborns urgently needed. Reproductive health 2014; 11: 82.

4. Black RE, Cousens S, Johnson HL, et al. Global, regional, and national causes of child mortality in 2008: a systematic analysis. Lancet 2010; 375(9730): 1969-87.

5. Caiulo VA, Gargani L, Caiulo S, et al. Lung ultrasound characteristics of community-acquired pneumonia in hospitalized children. Pediatric pulmonology 2013; 48(3): 280-7.

6. WHO Pocket book of hospital care for children, Guidelines for the management of common childhood illness. 2013. 
7. Saha D, Ronan A, Khan WA, Salam MA. Diagnosis of pneumonia in children with dehydrating diarrhoea. Journal of health, population, and nutrition 2014; 32(1): 14-8.

8. Singhi S, Dhawan A, Kataria S, Walia BN. Clinical signs of pneumonia in infants under 2 months. Archives of disease in childhood 1994; 70(5): 413-7.

9. Mathur NB, Garg K, Kumar S. Respiratory distress in neonates with special reference to pneumonia. Indian pediatrics 2002; 39(6): 529-37.

10. Smyllie HC, Blendis LM, Armitage P. Observer Disagreement in Physical Signs of the Respiratory System. Lancet 1965; 2(7409): 412-3.

11. Spiteri MA, Cook DG, Clarke SW. Reliability of eliciting physical signs in examination of the chest. Lancet 1988; 1(8590): 873-5.

12. Sarria E, Fischer GB, Lima JA, Menna Barreto SS, Flores JA, Sukiennik R. [Interobserver agreement in the radiological diagnosis of lower respiratory tract infections in children]. $J$ Pediatr (Rio J) 2003; 79(6): 497-503.

13. Bada C, Carreazo NY, Chalco JP, Huicho L. Inter-observer agreement in interpreting chest $\mathrm{X}$-rays on children with acute lower respiratory tract infections and concurrent wheezing. Sao Paulo medical journal $=$ Revista paulista de medicina 2007; 125(3): $150-4$.

14. Pauls S, Kruger S, Richter K, et al. [Interobserver agreement in the assessment of pulmonary infiltrates on chest radiography in community-acquired pneumonia]. RoFo : Fortschritte auf dem Gebiete der Rontgenstrahlen und der Nuklearmedizin 2007; 179(11): 1152-8.

15. Hamid M, Qazi SA, Khan MA. Clinical, nutritional and radiological features of pneumonia. JPMA The Journal of the Pakistan Medical Association 1996; 46(5): 95-9.
16. Bachur R, Perry H, Harper MB. Occult pneumonias: empiric chest radiographs in febrile children with leukocytosis. Annals of emergency medicine 1999; 33(2): 166-73.

17. Murphy CG, van de Pol AC, Harper MB, Bachur RG. Clinical predictors of occult pneumonia in the febrile child. Academic emergency medicine : official journal of the Society for Academic Emergency Medicine 2007; 14(3): 243-9.

18. World Health Organization(1990). Acute Respiratory Infections in Children: Case Management in Small Hospitals in Developing Countries. A Manual for Doctors and other Senior Health Workers. World Health Organization, Geneva.

19. World Health Organization (WHO) (1991) Technical Basis for the WHO Recommendations on the Management of Pneumonia in Children at First-level Health Facilities (WHO/ARI/91.20).

20. Cashat-Cruz M, Morales-Aguirre JJ, Mendoza-Azpiri M. Respiratory tract infections in children in developing countries. Seminars in pediatric infectious diseases 2005; 16(2): 84-92.

21. Duke T. Neonatal pneumonia in developing countries. Archives of disease in childhood Fetal and neonatal edition 2005; 90(3): F211-9.

22. Chisti MJ, Tebruegge M, La Vincente S, Graham SM, Duke T. Pneumonia in severely malnourished children in developing countries - mortality risk, aetiology and validity of WHO clinical signs: a systematic review. Tropical medicine \& international health : TM \& IH 2009; 14(10): 1173-89.

23. Palafox $\mathrm{M}$, Guiscafre $\mathrm{H}$, Reyes $\mathrm{H}$, Munoz $\mathrm{O}$, Martinez $\mathrm{H}$. Diagnostic value of tachypnoea in pneumonia defined radiologically. Archives of disease in childhood 2000; 82(1): 41-5. 